\title{
L'envers de la législation sur les voiles : une domestication de l'islam par la loi
}

\section{Franck Frégosi}

\section{(2) OpenEdition}

\section{Journals}

Édition électronique

URL : http://journals.openedition.org/rdr/930

DOI : $10.4000 /$ rdr.930

ISSN : 2534-7462

Éditeur

Presses universitaires de Strasbourg

\section{Édition imprimée}

Date de publication : 8 novembre 2016

Pagination : 83-106

ISBN : 978-2-86820-959-7

ISSN : 2493-8637

\section{Référence électronique}

Franck Frégosi, «L'envers de la législation sur les voiles : une domestication de l'islam par la loi », Revue du droit des religions [En ligne], 2 | 2016, mis en ligne le 11 février 2020, consulté le 19 novembre 2020. URL : http://journals.openedition.org/rdr/930 ; DOI : https://doi.org/10.4000/rdr.930

\section{(c) (†) (8)}

La revue du droit des religions est mise à disposition selon les termes de la Creative Commons Attribution - Pas d'Utilisation Commerciale 4.0 International - CC BY-NC 4.0. 


\section{L'ENVERS DE LA LÉGISLATION SUR LES VOILES : UNE DOMESTICATION DE L'ISLAM PAR LA LOI}

\section{Franck FRÉGOSI}

Institut d'études politiques d'Aix-en-Provence, CHERPA

\section{RÉSUMÉ}

Cet article propose une analyse sociologique des logiques d'action publique à l'œuvre dans l'élaboration de dispositifs législatifs ciblant des expressions vestimentaires réputées islamiques (loi du 15 mars 2004, loi du 11 octobre 2010). Il démontre que ces dispositifs s'inscrivent dans une logique globale de domestication de l'islam qui constitue la trame générale des politiques étatiques contemporaines ciblant les minorités religieuses et notamment l'islam en régime de laïcité. Il revient également sur le rôle clef de certains experts dans la production de grilles de lecture et de décryptage du port du voile intégral présenté comme une pratique reflétant une dérive sectaire de l'islam et jugée contraire à la compréhension modernisée de cette religion que les parlementaires souhaitent encourager.

\section{AbSTRACt}

This article proposes a sociological analysis of the logics of public action at work in the elaboration of legislative regulation of Islamic veiling (Act of 15 March 2004, Act of 11 October 2010). This article demonstrates that those regulations are part of a global logic of domestication of Islam, which constitutes the general scheme of the contemporary State policies toward Islam in the context of French laïcité. It also points out the central role of certain experts in production of keys for reading and deciphering on global veiling as a practice reflecting sectarian Islamic views and analysed as opposed to the modern understanding of this religion which the members of Parliament aim at encouraging. 


\begin{abstract}
À l'heure d'un État décrit comme recomposé ${ }^{1}$, c'est-à-dire notamment A dominé par une logique de gouvernance managériale et confronté à une pluralisation des sources d'autorité, le secteur religieux constitue l'un des secteurs de redéploiement de l'action étatique négligé par l'analyse des politiques publiques.
\end{abstract}

En l'espèce, force est pourtant de constater l'existence d'un véritable paradoxe illustré par le décalage entre le discours dominant des élites politiques hexagonales qui tend à consacrer l'extériorité absolue du fait religieux par rapport à l'administration publique - et plus largement par rapport au fonctionnement régulier de l'État - comme clef de voûte de l'édifice républicain et laïque d'une part, et d'autre part la réalité de multiples processus d'interactions sociales, institutionnelles, voire même de prolongements juridiques (vote de lois sur le port du voile dans l'école publique et dans l'espace public) entre la sphère étatique et la sphère religieuse. Longtemps demeuré un point aveugle de l'analyse des politiques publiques ${ }^{2}$ et de la sociologie de l'action publique ${ }^{3}$, la prise en compte du champ religieux à la fois comme un lieu effectif d'affirmation de l'action publique, mais aussi comme un secteur stratégique d'élaboration de politiques publiques ciblées visant notamment des groupes religieux minoritaires (groupes musulmans) et certaines expressions religieuses socialement controversées (sectes), s'est imposée comme une donnée sociologique incontournable. La plupart du temps, l'intervention de la puissance publique dans l'espace du religieux s'effectue en lien plus ou moins direct avec les autres secteurs de déploiement de l'action publique que sont la politique de sécurité, la politique migratoire, la politique de la ville, la politique étrangère, la politique scolaire, etc.

Qu'il s'agisse de pointer les diverses facettes religieuses de la politique sécuritaire (mise sur pied de processus ciblés de déradicalisation et de contre-radicalisation ${ }^{4}$, contrôle des flux d'imams étrangers ${ }^{5}$, systématisation

1. Le Galès P., Vezinat N. (dir.), L’État recomposé, Paris, PUF, 2014.

2. Muller P., Les politiques publiques, Paris, PUF, 2010. - Kubler D., Maillard J. DE, Analyser les politiques publiques, Grenoble, PUG, 2009. - Boussaguet L., Jacquot S., Ravinet P. (dir.), Dictionnaire des politiques publiques, Paris, Presses de Sciences Po, $2^{\mathrm{e}}$ éd. 2006.

3. Lascoumes P., Le Galès P., Sociologie de l'action publique, Paris, Armand Colin, 2009. - Gaudin J.-P., L'action publique : sociologie et politique, Paris, Presses de Sciences Po/ Dalloz, 2004.

4. CONESA P., Quelle politique de contre-radicalisation en France?, rapport pour la Fondation d'aide aux victimes du terrorisme (FAVT), déc. 2014.

5. Jounnneau S., «L'imam, clerc sans clergé ni église : les répertoires d'une autorité dissimulée dans les cadres de l'interaction », Genèses, n⿳ 88, 2012, p. 6-24; " "Ne pas perdre la foi dans l'imamat". Comment se maintiennent les "vocations" d'imams bénévoles en 
de formations civiques destinées aux cadres religieux musulmans ${ }^{6} \ldots$ ) ou l'existence de dispositifs magnifiant le caractère réputé incontournable du dialogue interreligieux et des questions religieuses en matière de relations internationales ${ }^{7}$, sans parler des politiques valorisant l'expérience française de laïcisation à destination de l'étranger, il n'y a plus lieu de douter un seul instant que le religieux est bien inscrit à l'agenda public de cette République réputée laique.

Cet article vise à mettre à nu l'un des aspects de la fabrication d'un problème public autour du fait islamique à partir de l'analyse des intentions et des logiques d'action publique à l'œuvre dans l'élaboration de dispositifs législatifs ciblant des expressions vestimentaires réputées islamiques ${ }^{8}$.

Outre l'identification d'une logique de domestication de l'islam (1) qui constitue la trame générale des politiques étatiques contemporaines ciblant l'islam sui generis, et notamment illustrée par les votes successifs de deux lois (2) encadrant la visibilité publique du voile dans la sphère publique (loi du 15 mars 2004) et dans l'espace public (loi du 11 octobre 2010), il s'agit aussi dans cet article de s'intéresser aux divers types de référentiels mobilisés afin de légitimer ces législations limitant le port de tenues vestimentaires islamiques. Ce faisant, une attention particulière sera accordée aux acteurs impliqués dans la production de discours experts sur la problématique du voile intégral (3) sur lesquels les parlementaires ont pu s'adosser afin de légitimer socialement l'adoption de législations limitant la visibilité publique de comportements islamiques dénoncés comme déviants par rapport à une acception réputée républicaine de l'islam.

France », Sociétés contemporaines, n 84, 2011, p. 103-125; "Régulariser ou non un imam étranger en France : droit au séjour et définition du "bon imam" en pays laïque », Politix, n 86, 2009, p. 147-166.

6. Messner F., Zwilling A.-L. (dir.), Formation des cadres religieux en France. Une affaire d'État?, Genève, Labor et Fides, 2010.

7. Lacorne D., Vaîsse J., Willaime J.-P., La diplomatie au défi des religions. Tensions, guerres, médiations, Paris, O. Jacob, 2014. - BALAS M., « Un pluralisme sans conflits. Sant'Egidio : diplomatie et religion », Terrain, sept. 2008, p. 51-60.

8. Il n'entre pas dans notre propos de nous prononcer sur l'islamité canonique desdites tenues, aussi nous bornons-nous à les qualifier d'islamiques par référence au simple fait que celles qui les portent les qualifient comme telles, ou plus simplement parce qu'elles relèvent d'un univers de pratiques et d'usages en vigueur dans le monde musulman. 


\section{LA DOMESTICATION DE L'ISLAM DANS TOUS SES ÉTATS}

Les prémices de ma réflexion en termes de domestication de l'islam s'inspirent de celle menée par Thijl Sunier de l'Université libre d'Amsterdam. Dans une conférence de novembre $2009^{9}$, cet anthropologue spécialiste de l'islam s'interrogeait sur les méfaits académiques d'une systématisation des débats publics focalisés exclusivement sur les enjeux sécuritaires et politiques à propos de l'islam en Europe. Il en profitait pour souligner combien la problématique lancinante de la quête indéfinie d'un islam européen pouvait être perçue comme la résultante d'enjeux sécuritaires et identitaires parmi lesquels figure en bonne place la question de la domestication de l'islam.

L'idée de domestication ${ }^{10}$ suggère un processus d'apprivoisement par l'imposition de règles, de comportements, d'attitudes requises en réponse à une sollicitation extérieure émanant d'un maître, en tout cas d'un dominant adoptant une position de surplomb par rapport à un dominé. La domestication est en somme, dans le monde animalier, peu ou prou l'équivalent du processus de normalisation dans le monde des humains, entendu comme la délimitation de règles et leur imposition visant à construire objectivement une ligne de démarcation entre l'univers de l'anormalité sociale et celui du monde normé ou plus exactement du monde orthonormé. C'est notamment dans ce sens que le vocable se retrouve dans le lexique des sciences sociales, aussi bien dans les travaux de Michel Foucault sur les mécanismes de construction sociale de l'anormalité à partir de l'utilisation des expertises médicales et psychiatriques ${ }^{11}$, que plus en amont dans ceux de Max Weber sur la problématique de la domination ${ }^{12}$. Le processus de domestication participe d'une microphysique du pouvoir pour l'un, d'une sociologie systématique du pouvoir pour l'autre, c'est-à-dire d'une analyse des procédures visant à consolider l'asymétrie sur laquelle repose toute relation de domination entre des individus inégalement dotés en matière de ressources techniques, sociales

9. SuniER T., Beyond the domestication of Islam in Europe, Vrije Universiteit Amsterdam, Faculteit der Sociale Wetenschappen, 2009.

10. Étymologiquement, le vocable de domestication construit à partir du latin domesticus, littéralement « de la maison », désigne habituellement le processus au terme duquel un animal réputé sauvage va peu à peu intégrer le voisinage immédiat des hommes et évoluer dans l'entourage de ces derniers. La domestication suggère alors un processus de dressage d'un animal afin qu'il obéisse et se conforme à la tâche ou au service attendu de lui.

11. Foucault M., Les anormaux. Cours au Collège de France (1974-1975), Paris, Gallimard/ Le Seuil, 1999.

12. Weber M., La domination, Paris, La Découverte, 2013. 
ou symboliques et occupant dans la société des positions sociales pour les uns dominantes, pour les autres dominées.

La domestication renvoie donc aussi à un processus d'intériorisation de normes, d'assimilation d'attitudes, de pratiques et de comportements attendus des individus dominés dans un environnement déterminé afin d'assurer leur pleine insertion dans ledit environnement, en d'autres termes leur participation à l'illusio collectif sur lequel repose la société, et éviter toute forme de sécession ${ }^{13}$. En cela la domestication s'apparente à un processus de socialisation dans le sens où elle présuppose des apprentissages successifs, des répétitions, l'existence de modèles à suivre et de rôles sociaux à respecter. Elle s'en sépare toutefois dans le fait que la socialisation d'un individu vise prioritairement à lui permettre de participer pleinement à la vie d'une société, à lui faire acquérir les clefs et les codes du milieu dans lequel il va évoluer, de tisser de nouvelles relations et de cheminer socialement dans un ensemble plus vaste dont il devient un des acteurs, là où l'idée de domestication tend davantage à consacrer un rapport de subordination entre ceux qui formulent les normes et ceux auxquels elles s'imposent, comme si leur pleine insertion dans la société était conditionnée, assujettie à certains renoncements, à certaines limitations publiques énoncées.

L'idée de domestication à laquelle nous nous référons suggère en amont l'incapacité des dits dominés de parvenir par eux-mêmes à trouver leur place légitime dans la société en raison de singularités, de particularités dont ils seraient porteurs. Il leur est dès lors suggéré, par les tenants de la domestication, de consentir à se soumettre à ce processus afin de se « libérer » des supposées entraves qui empêchent leur pleine insertion dans la société.

Le phénomène de domestication de la religion s'enracine plus globalement dans un processus historique assez classique de mise au pas des religions par l'imposition d'un cadre et d'un schéma d'organisation ou de refonte de l'organisation du culte, par le vote de lois encadrant la visibilité sociale des symboles religieux dans la cité et la production d'une version officielle de ladite religion censée être compatible avec la société environnante. C'est ainsi qu'un tel processus avait déjà été observé durant la Révolution française à l'encontre du catholicisme, avec l'élaboration de la Constitution civile du clergé en 1790, comme il a ensuite concerné le judaïsme métropolitain et

13. Bourdieu P., Sur l'État. Cours au Collège de France (1989-1992), Paris, Seuil/Raisons d'agir, 2012. 
celui d'Algérie au cours du XIX ${ }^{e}$ siècle ${ }^{14}$ et, de façon plus ou moins large, l'islam durant la période coloniale en Algérie ${ }^{15}$. La précédente tentative de domestication de l'islam en contexte colonial avait surtout abouti à l'invention du culte musulman, là où auparavant il n'était question que d'islam sui generis, et à la mise sur pied d'une classification administrative des édifices du culte et du personnel des desservants nommés et rétribués par l'État colonial.

Or, le processus contemporain de domestication de l'islam qui se déroule sous nos yeux, loin de se résumer à une question de réorganisation du culte (création du Conseil français du culte musulman, CFCM et des conseils régionaux, $\mathrm{CRCM}$ ), consiste davantage en l'imposition indirecte de normes encadrant la visibilité sociale et urbaine de l'islam. La façon dont ces normes contraignantes ont vu le jour et leur mode de légitimation semblent démontrer qu'elles n'ont pas formellement vocation à permettre aux musulmans de gravir les échelons de la société à laquelle ils vont s'agréger, d'acquérir des droits nouveaux, mais bien de les inciter à rester à leur « juste» place, répondant avant tout à un désir de remodelage de l'islam afin de répondre aux demandes et aux angoisses de la société environnante par rapport à une présence religieuse jugée déstabilisante. S'ajoute à cela le fait que, bien qu'entré dans le moule qui lui a été imposé, passé sous les fourches caudines de la loi, cet islam soumis restera toujours suspecté de n'être pas totalement maîtrisable, et devra donc toujours être tenu en respect comme si son altérité intrinsèque, tel l'état sauvage d'un animal domestiqué, pouvait à tout moment resurgir.

Au regard de l'actualité récente, à l'occasion des drames sanglants qui ont frappé Paris et sa périphérie durant l'année 2015, comment ne pas songer à l'injonction contradictoire faite régulièrement aux musulmans de France de devoir se désolidariser collectivement, de façon communautaire, des agissements criminels d'individus musulmans se réclamant de Daech, alors qu'habituellement reproche leur ait fait de donner trop prise à des revendications dites communautaristes. Il se dégage de ce type d'appel l'impression que l'islam et les musulmans sont toujours socialement en sursis, et qu'ils doivent toujours donner des gages, des preuves de leur « bonne intégration » au sein d'une société qui est pourtant aussi la leur, mais dans laquelle leur présence semble alimenter bien des visions obsessionnelles ${ }^{16}$.

14. Assan V., Les consistoires israélites d'Algérie au XIX siècle. L'alliance de la civilisation et de la religion, Paris, Armand Colin, 2012.

15. SAAÏDIA O., Algérie coloniale : musulmans et chrétiens : le contrôle de l'État (1830-1914), Paris, CNRS Ed., 2015.

16. V. les essais d'A. Finkielkraut, de R. Camus, le dernier roman de M. Houellebecq ou les fulminations d'É. Zemmour. 
Fort de ces prémices, je me suis demandé dans quelle mesure l'intuition de ce collègue néerlandais, par-delà la question de la récurrence du prisme sécuritaire comme mode de gestion à la fois le plus ancien du fait islamique en France et le plus persistant, ne devait pas nous amener à reconsidérer nos analyses à propos de la régulation publique du fait islamique. Celle-ci tendait jusque-là à être analysée comme un banal processus de normalisation de l'islam combinant la nécessité de "meubler» l'interface entre l'État et les groupes musulmans par des organismes représentatifs (CORIF, Conseil représentatif des musulmans de France, $\mathrm{CFCM}^{17}$ ) censés d'une part théoriquement relayer le point de vue des intérêts du culte musulman et de ses fidèles auprès des pouvoirs étatiques, et veiller ensuite à ce que les droits cultuels des fidèles fassent l'objet d'un traitement public équivalent à ceux des autres groupes religieux.

Mais il apparaît également, à l'aune de l'inflation législative ayant l'islam pour cible et les nombreux débats y afférents, qu'il y a aussi matière à raisonner en termes de processus de formatage d'un islam dit " républicain » censé accepter par lui-même, ou, le cas échéant, de se voir imposer des normes encadrant de façon limitative non seulement sa visibilité sociale (par l'exclusion du voile simple de l'école publique et du voile intégral de l'espace public), mais aussi ses modes d'expressions, par l'injonction faite par médias et intellectuels interposés de promouvoir un islam moderne, un « islam pour notre temps ${ }^{18}$. Il s'agit plus largement de pointer la propension forte, en régime de laïcité proclamée, de vouloir durablement modeler un religieux sur mesure, un islam «made in France » et lui apposer une sorte d'appellation d'origine contrôlée républicaine (AOCR).

Par domestication de l'islam, il convient donc d'entendre un processus de gouvernance global par lequel les pouvoirs publics tendent, sous couvert de favoriser une pleine intégration de l'islam dans la réalité des sociétés européennes, de mettre sur pied des politiques ciblées visant à endiguer et encadrer de façon étroite la visibilité sociale de l'islam et au besoin la pratique publique de celle-ci. Il s'agit en fait de produire, développer et déployer techniquement des dispositifs normatifs, législatifs et réglementaires destinés à contrôler l'expression publique de ladite religion dans la cité séculière.

Ce contrôle peut porter principalement sur les aspects externes, sur les manifestations visibles de la religion (voile, lieux de culte, nourriture,

17. Frégosi F., L’islam dans la laicité, Paris, Pluriel, 2011.

18. Bidar A., Un islam pour notre temps, Paris, Seuil, 2004. 
jeûne...) ainsi que sur leurs supports institutionnels (associations, organes représentatifs...). Mais ledit contrôle peut également se prolonger de façon plus indirecte par une surveillance étroite des dimensions plus internes de la foi que sont les croyances et les représentations religieuses (favoriser les formes réputées modernes de l'islam, former des imams, dénoncer les dérives salafistes comme sectaires et contraires à l'islam...).

Le processus de domestication de l'islam en France renvoie donc à une logique d'action publique centrée sur le contrôle optimal de la religion musulmane et la production d'un islam sinon officiel, en tout cas jugé compatible avec les idéaux de la République, et partant de là, sur la relégation d'autres expressions de l'islam dans les confins de l'archaïsme, de l'extrémisme et du fanatisme. En plein débat sur le voile intégral, le député Jean Glavany avait par exemple estimé qu'il convenait de mener contre le salafisme, cette version réputée sectaire et exclusiviste de l'islam, une guerre sans fin au même titre que la République s'est dressée contre le nazisme et le fascisme.

\section{LA DOMESTICATION LÉGISLATIVE DE L'ISLAM}

La domestication de l'islam ne saurait toutefois se résumer à un processus de négociations permanentes entre des opérateurs publics désireux de procéder à une rationalisation du nombre de leurs interlocuteurs musulmans et des notables musulmans soucieux de voir confirmer leur hégémonie sur le champ islamique hexagonal et d'apparaître comme les partenaires les plus loyaux des pouvoirs publics. La domestication de l'islam doit également être appréhendée comme un processus qui vise à modifier profondément les attitudes, les comportements sociaux comme les pratiques supposés caractériser ceux (les musulmans) auxquels elle s'applique, afin de favoriser leur agrégation à la société environnante. L'objectif implicite est alors d'influer de façon plus ou moins contraignante sur les contours et la visibilité sociale de l'islam afin d'en gommer les «éventuelles » aspérités sociales (indistinction du cultuel et du culturel, étendue des prescriptions religieuses et des interdits, indifférenciation entre le politique et le religieux, inégalité entre hommes et femmes, visibilité dans l'espace public...), afin d'œuvrer à son insertion durable dans la société.

Cette domestication de l'islam, déjà à l'œuvre dans la tentative de centralisation de la représentation du culte autour du CFCM à l'échelon national et des CRCM à l'échelon des régions, est bien plus explicite si on prend la 
peine d'analyser cette fois la façon dont le fait islamique est appréhendé par divers textes de loi relatifs au port du voile, faisant suite à des rapports officiels tels ceux de la commission Stasi en 2003 et de la mission sur le voile intégral de l'Assemblée nationale en $2010^{19}$. Ces deux initiatives devaient contribuer plus largement, derrière la politisation du voile ${ }^{20}$, à la diffusion de cadres et de grilles d'analyse au travers desquels devait être appréhendée la réalité sociale de l'islam en France, ses dynamiques internes comme les éventuels points de tensions supposés croissants entre la société hexagonale et certains comportements islamiques.

La lettre de mission du 3 juillet 2003 créant la commission Stasi censée réfléchir à l'application du principe de laïcité dans la République mentionnait que ladite commission devait plus spécifiquement répondre à la question suivante : "Comment donner toute la force au sein de l'école publique à l'exigence de laïcité ? ${ }^{21}$. Son champ d'investigation s'est en fait avéré bien plus vaste. Outre les personnels de l'Éducation nationale, ladite commission a auditionné des représentants des confessions, des familles de pensée, ainsi que des partenaires sociaux, des élus, des professionnels du secteur hospitalier et des chefs d'entreprise. Le rapport donna ainsi le sentiment que c'était l'ensemble de la société qui était confronté à une intrusion massive du religieux dans des secteurs auparavant largement laïcisés (école, santé, travail, sport...), menaçant ainsi la cohésion sociale de la société tout entière. « Or, c'est une des constatations, écrit Bernard Stasi, que nous avons faites tout au long de nos travaux, et pour certains d'entre nous avec étonnement, les comportements, les agissements attentatoires à la laïcité sont de plus en plus nombreux, en particulier dans l'espace public. ${ }^{22}$ Bernard Stasi, conscient des effets dépréciatifs induits par un tel constat, prit toutefois la peine de nuancer cette impression générale d'une société menacée dans ses fondements par un islam de plus en plus visible ; il déclara avoir « regretté la polarisation excessive, et parfois exclusive, sur le foulard islamique ${ }^{23}$, reconnaissant que «le repliement communautariste est plus subi que voulu» ${ }^{24}$ et lié à

19. Rapport d'information sur la pratique du port du voile intégral sur le territoire national, Paris, Assemblée nationale, 26 janv. 2010, nº 2262.

20. LorCerie F. (dir.), La politisation du voile : l'affaire en France, en Europe et dans le monde arabe, Paris, L'Harmattan, 2005.

21. Rapport de la commission de réflexion sur l'application du principe de laïcité dans la République remis au Président de la République le 11 décembre 2003, Paris, La Documentation française, 2003, p. 3.

22. Ibid., p. 6.

23. Ibid.

24. Ibid., p. 45. 
des conditions sociales et urbaines davantage qu'à la supposée essence de l'islam. En dépit de ses ultimes précautions et de sa claire dénonciation d'une montée d'une haine envers l'islam, c'est bien autour de la question du voile que devaient néanmoins se focaliser tous les commentaires.

D'ailleurs, de toutes les propositions émises par la commission ne fut retenue que celle d'interdire par la loi les signes religieux réputés ostensibles. Oubliée l'idée d'instaurer dans les écoles publiques deux fêtes, une juive et une musulmane (Kippour et Aïd El Kébir), de permettre aux salariés de choisir une de ces fêtes religieuses sur leur crédit de jours fériés, ou de créer une école nationale d'études islamiques. Le législateur ne retint que la suggestion de légiférer contre le port de signes religieux, et en l'état contre le voile dans l'école publique. Les quelques cas d'élèves sikhs arborant des turbans ou les allusions ministérielles à propos de grandes croix assyriennes ne devaient pas faire illusion, c'est bien le voile en tant que symbole islamique qui était visé. De la même façon que la commission admettait dans son écrasante majorité (abstention de Jean Baubérot) le principe de l'interdiction des signes dits ostensibles (croix, kippa, foulards), ses membres durent énumérer en retour ce qui à leurs yeux étaient des signes non ostensibles, donc socialement légitimes et acceptables dans l'enceinte scolaire. "Ne sont pas regardés comme des signes manifestant une appartenance religieuse les signes discrets que sont par exemple médailles, petites croix, étoiles de David, mains de Fatimah, ou petits Coran $»^{25}$. Oui au colifichet ethnique (main de Fatimah), non au foulard réputé islamique, tel pourrait résumer le point de vue des membres de la commission. Si le rapport Stasi ne reprit pas explicitement à son compte l'idée d'une islamisation générale de la société française, le rapport de la mission parlementaire sur le port du voile intégral fut nettement plus explicite. À l'occasion des auditions, le député André Gerin, à l'origine de la création de cette mission d'information parlementaire, évoquera clairement à plusieurs reprises l'islamisation de la société et de l'Europe dont le port du voile intégral serait la manifestation.

Si tous les gouvernements européens aspirent peu ou prou à dialoguer prioritairement avec des musulmans réputés «modérés» ou " modernes », la France est sans nul doute l'un des États (avec la Confédération helvétique et la Belgique) dans lequel la puissance publique elle-même, via l'outil législatif, participe activement à cette tentative de délimitation des contours de l'islam réputé moderne. L'implicite est des plus simples: si les musulmans veulent pouvoir jouir d'un traitement équivalent à celui dont bénéficient les autres

25. Ibid., p. 68. 
cultes, ils doivent en amont se délester de certains usages présentés comme islamiques, ou comme le déclarait le philosophe Abdennour Bidar, auditionné par la mission d'information sur le port du voile intégral, entreprendre une « cure d'amaigrissement» ou se la voir imposer par l'État par le vote de lois encadrant la visibilité sociale de l'islam. On peut renvoyer sur ce point à l'échange entre le député socialiste Jean Glavany, membre de la mission Gerin, et Mohamed Moussaoui, président du CFCM, lors de son audition devant la mission d'information sur le voile intégral. Le député, manifestement agacé par le fait que cet interlocuteur rechignait à dénoncer la pratique du voile intégral comme l'expression d'un islam intégriste, devait déclarer : «Si vous ne nous aidez pas Messieurs, il faudra bien que nous vous aidions à nous aider... $»^{26}$. Ce propos direct trahit l'idée que l'on serait face à une version islamocentrée de l'intention qui était déjà celle des hommes des Lumières et des révolutionnaires français lorsqu'ils souhaitaient œuvrer à l'émancipation des juifs de France ${ }^{27}$ et parallèlement à leur régénération physique, morale et politique conceptualisée par l'abbé Grégoire ${ }^{28}$. Il s'agissait à l'époque de permettre une pleine accession à la citoyenneté des israélites de France (dont la présence n'était jusque-là que tolérée), tout en les conduisant sur les voies de l'émancipation civile par rapport à la dimension ethnique, communautaire sous-tendue par la judéité. "Il faut tout refuser aux juifs comme nation, et accorder tout aux juifs comme individus » avait déclaré à la tribune de l'assemblée Clermont-Tonnerre en 1789. À l'époque, il s'agissait d'instaurer une réelle égalité de droits sans distinction de confession, de conférer aux juifs les droits civils dont ils ne bénéficiaient pas sur l'ensemble du territoire et de faire tomber les réserves juridiques qui s'imposaient à eux. Cela fut fait avec le décret du 27 septembre 1791 et rendu effectif plus tardivement.

Aujourd'hui, la problématique à propos des musulmans est quelque peu différente. Même s'il est parfois question de l'émancipation des musulmans et surtout des musulmanes (à propos du voile), il s'agit objectivement moins de les faire bénéficier de droits nouveaux, mais plutôt de suggérer que des devoirs nouveaux leur soient imposés, afin de les rendre plus "conformes " à ce qu'ils devraient être. L'idée est notamment d'opérer de telle façon que les musulmans s'engagent collectivement dans la voie d'une reformulation de leur religion à partir d'une pratique de plus en plus sélective de celle-ci (abandon $\mathrm{du}$ voile dans les écoles publiques, interdiction du voile intégral dans l'espace

26. Rapport d'information..., op cit., p. 395.

27. Winock M., La France et les Juifs : de 1789 à nos jours, Paris, Seuil, 2004.

28. BAdinter R., Libres et égaux : l'émancipation des Juifs (1789-1791), Paris, Fayard, 1989. 
public, limitation du recours à l'abattage rituel ${ }^{29} \ldots$... Le cas échéant, le législateur peut leur «prêter » main-forte ou, pour être plus précis, leur forcer la main par la production de règles nouvelles contraignantes. Cela fut le cas avec la loi du 15 mars 2004 sur les signes religieux dans l'école publique et plus récemment avec la loi du 11 octobre 2010 interdisant dans l'espace public le voile intégral. Ces deux lois entendaient délimiter de façon restrictive l'espace de manifestation de la disparité religieuse. Lors des auditions, le député André Gerin prétendait vouloir « aider » les musulmans à se doter d'un « islam républicainement compatible », version actualisée d'un "islam régénéré ». Le député UMP Éric Raoult, rapporteur de la mission, avait pris lui-même le soin de recourir au vocable de "loi de libération » à propos de la future loi interdisant le voile intégral. On est donc bien là face à un schéma d'émancipation décrétée par le haut, d'une domestication par la loi. En l'état, la domestication consiste à atténuer, à gommer les éléments jugés trop voyants et supposés induire une trop grande disparité avec le reste de la société. C'est là une vieille idée qui vise à inciter la religion à se délester de certains de ses particularismes et notamment de pratiques extérieures, pour devenir une religion épurée davantage adaptée à la vie démocratique.

Tel était déjà l'avis d'Alexis de Tocqueville lorsqu'il décrivait dans De la démocratie en Amérique la façon dont à partir du cas américain, les religions devaient jouer un rôle actif dans les sociétés démocratiques en se cantonnant à la sphère du culte et à distance du politique (principe de séparation des cultes et de l'État), mais aussi en rognant sur certaines de leurs pratiques. « Une autre vérité me paraît fort claire : c'est que les religions doivent moins se charger de pratiques extérieures dans les temps démocratiques que dans tous les autres. ${ }^{30}$ Tocqueville prenait l'exemple du catholicisme minoritaire sur le continent nord-américain, replié sur les lieux de culte et dépourvu de pratiques extérieures telles que les processions ou les chemins de croix sur les routes.

Pour Jean Glavany (qui s'abstiendra de voter la loi !), l'interdiction du voile intégral devait être motivée par la volonté de lutter contre une pratique décrite comme sectaire et politique, et non par le désir de limiter l'exercice public du culte musulman dans l'espace public :

29. En pleine polémique lancée par Marine Le Pen sur l'abattage rituel en Île-de-France, François Fillon n'avait pas hésité à inviter juifs et musulmans à renoncer à cette pratique jugée archaïque.

30. Tocqueville A. De, De la démocratie en Amérique. II, Paris, Garnier-Flammarion, 1981, p. 34 . 
«[...] les responsables du Conseil Français du Culte Musulman, que nous avons rencontrés longuement, nous ont expliqué que cette pratique n'était nullement exigée par le Coran. [...] on a repéré que c'étaient des pratiques extrémistes, intégristes, et donc très politiques, qui étaient aux sources de la pratique du voile intégral. Il y en avait deux, la pratique des Talibans, en Afghanistan, et puis des Salafistes wahhabites en Arabie Saoudite. Et donc il y a là deux sources qui n'ont rien à voir avec le Coran, et donc l'Islam, qui sont des pratiques minoritaires, extrémistes, sectaires et très politiques. Donc c'est à ce titre-là qu'on les combat, au même titre que l'on combat d'autres idéologies, et pas au nom de la laïcité. ${ }^{31}$

Le député s'adossant à l'avis du CFCM qui considérait que le niqab n'était pas requis par le Coran, estima que par conséquent le choix des députés ne visait en rien l'islam comme religion, mais bien une expression déviante de celui-ci.

On est là au coeur d'un autre paradoxe qui veut que des députés en viennent cette fois à s'adosser à une exégèse du texte coranique afin de légitimer indirectement leur démarche législative de prohibition d'une tenue portée par des femmes musulmanes. De fait, le législateur est implicitement invité à se transformer en avocat séculier, mais non moins zélé, d'une compréhension réputée "équilibrée » de la religion susceptible d'avoir droit de cité dans la cité laïque.

La France est bien devenue le théâtre privilégié d'une mise en scène des rapports entre l'islam entendu comme fait religieux et une certaine conception exclusiviste de la République ${ }^{32}$ sous la forme d'une tension permanente entre le principe du libre exercice du culte et la tendance à vouloir peser en profondeur sur le contenu intrinsèque de cette religion. Organiser le culte ou réformer la religion, tel est bien le dilemme auquel ont été constamment confrontés les pouvoirs depuis une vingtaine d'années s'agissant de l'islam.

31. Frégosi F., Kosulu D., Challenges of religious accommodation in family law and labor, regulation of public space and public funding (French socio-legal report), RELIGARE Project, European Commission/ $7^{\text {th }}$ Framework Programme, 29 Oct. 2013, p. 79.

32. Pour une analyse critique, V. LABORDE C., Français, encore un effort pour être républicains !, Paris, Seuil, 2010 et SiEFFERT D., Comment peut-on être (vraiment) républicain ?, Paris, La Découverte, 2006. 


\section{DU BON USAGE DE CERTAINES EXPERTISES... OU DE LA DIFFICILE DÉLIMITATION ENTRE EXPERTISE ET DISCOURS MILITANT}

L'analyse des modalités de domestication de l'islam passe par la prise en compte de l'implication dans ce processus non seulement de certains opérateurs religieux musulmans ${ }^{33}$, mais aussi et surtout par la diffusion de grilles d'analyse et de dispositifs discursifs produits par des experts pouvant légitimer socialement les choix législatifs des parlementaires.

La domestication de l'islam peut donc découler de l'autolimitation ou de l'autodiscipline que des opérateurs musulmans s'imposent à eux-mêmes de façon plus ou moins consciente. Ceux-ci consentiraient, par exemple, en matière d'observances, à promouvoir une vision supposée moins extensive de la religion, à moduler dans le sens de la discrétion leurs demandes religieuses, pouvant aller jusqu'à une limitation du processus même d'extériorisation de la foi et à un abandon de signes de visibilité comme le port du voile simple, quand ils ne dénoncent pas directement le recours au voile intégral.

Je souhaiterais toutefois faire porter mon analyse sur le rôle joué par certains experts dans la production de grilles de lecture et d'analyse sur les personnes arborant le port du voile intégral qui ont par la suite été abondamment reprises par les parlementaires pour légitimer l'interdiction généralisée de tenues dissimulant le visage, dans l'intérêt, selon eux, de l'islam et des musulmans.

Soucieux de se dédouaner par rapport à l'accusation de surfer sur l'islamophobie ambiante et de chercher à légiférer sur la pratique du culte musulman, il apparaissait indispensable aux parlementaires de chercher à légitimer leur décision en s'adossant à des analyses démontrant paradoxalement que la pratique du voile intégral n'avait non seulement rien de religieux, mais pouvait même apparaître comme contraire, comme une déviation par rapport à la religion musulmane elle-même.

«Comment condamner cette pratique du voile intégral, peut-on en effet lire en introduction du rapport de la mission d'information, et la prohiber sans donner le sentiment que l'on s'attaque à une partie de la population en raison de ses origines ou de sa confession ? Il ressort de nos auditions que le port du voile intégral n'est pas une prescription de

33. V. FrÉGOSI F., « Domestiquer la religion en régime de laïcité : le cas de l'islam en France, in Zuber V., Cabanel P., Liogier R. (dir.), Croire, s'engager, chercher. Autour de Jean Baubérot, du protestantisme à la laïcité, Turnhout, Brepols, 2016, p. 221-242. 
l'islam [en gras dans le texte] mais une pratique culturelle pour certains et militante pour d'autres : mais il est apparu de manière tout aussi évidente que notre République devait fortement encourager, conforter les représentants et les responsables de l'islam de France pour qu'ils puissent prendre toute leur part dans la lutte contre ces pratiques radicales qui donnent une image déformée de cette religion. ${ }^{34}$

Parmi les personnalités consultées, on se doit de distinguer celles qui l'ont été au titre de leurs compétences avérées sur l'islam ou le monde musulman (universitaires, chercheurs, enseignants...), les administrateurs du CFCM et des CRCM, les responsables musulmans impliqués dans la gestion des lieux de culte, les présidents d'associations musulmanes locales et des figures musulmanes intellectuelles réputées (Tariq Ramadan). Figuraient également des personnalités ayant rang d'experts ${ }^{35}$, s'étant illustrées par des essais et des tribunes dans la presse dénonçant la pratique du voile intégral comme anachronique et contraire à une vision résolument moderne de la religion musulmane qu'il convenait de promouvoir. C'est vers elles que nous avons choisi d'orienter notre recherche en étudiant plus spécifiquement les interventions et les rôles joués par Dounia Bouzar et Abdennour Bidar en matière de production d'un discours expert sur le port du voile intégral et la nécessité d'en limiter la pratique.

Ces deux personnalités fréquemment sollicitées par les médias lors des controverses relatives à l'islam furent non seulement les premières à être auditionnées par la mission d'information, mais leurs témoignages se sont avérés décisifs, comme en atteste la reprise en boucle de leurs analyses critiques (mais pas nécessairement convergentes) par rapport au voile intégral et ce qu'il serait censé véhiculer (une vision déformée de l'islam pour l'une, l'expression d'un différentialisme inacceptable pour l'autre). C'est ainsi que les membres de la mission d'information reprirent volontiers à leur compte les analyses proposées par ces deux experts comme autant de certitudes quant à la nature sectaire et intégriste du voile intégral d'une part (3.1), et à la nécessité d'œuvrer à l'avènement d'un islam modernisé par la prohibition de cet usage vestimentaire d'autre part (3.2).

34. Rapport d'information..., op. cit., p. 23.

35. Outre D. Bouzar et A. Bidar, fait aussi partie de cette catégorie d'experts : Antoine Sfeir, directeur de la revue Les Cahiers de l'Orient, membre fondateur de l'Observatoire international de la laïcité contre les dérives communautaires et invité régulier de l'émission « C dans l'air » sur France 5. 


\subsection{LE VOILE INTÉGRAL, EXPRESSION D'UNE DÉVIANCE SECTAIRE}

C'est à Dounia Bouzar que revint l'honneur d'être la première personnalité auditionnée par la mission d'information parlementaire sur la pratique du port du voile intégral sur le territoire national. Lors de son audition, elle fut présentée par le député André Gerin comme une anthropologue du fait religieux ayant travaillé sur la question du voile à l'école publique et ayant ensuite publié un article assimilant la pratique du port du voile intégral à l'expression d'une dérive sectaire.

« Vous avez récemment, déclara le député du Rhône, pris une position très claire à propos du voile intégral en expliquant que la burqa ou le niqab n'était pas un signe religieux, mais le produit d'une dérive sectaire. Selon vous, il ne faut pas aborder le problème du voile intégral en le reliant à l'islam, car cela reviendrait à valider la démarche de ces mouvements sectaires qui appellent au port de ce type de voile et à renforcer leur autorité. ${ }^{36}$

En 2004, Dounia Bouzar s'était élevée contre le vote de la loi proscrivant le port du foulard ou du voile dit islamique par des collégiennes et des lycéennes au sein des établissements publics d'enseignement (primaire et secondaire) ${ }^{37}$. Elle avait même publié, avec une jeune femme musulmane, chef d'entreprise, militante associative voilée, Saida Kada, un livre témoignage à deux voix ${ }^{38}$ dans lequel elle démontrait notamment que le port de ce voile, loin de traduire une mise en retrait de la femme voilée du reste de la société, pouvait apparaître a contrario comme un vecteur d'implication sociale et citoyenne. Dounia Bouzar est une ancienne éducatrice à la Protection judiciaire de la jeunesse (PJJ), titulaire d'une thèse en anthropologie soutenue en 2006 sous la direction d'Olivier Roy. Ancienne membre (nommée) du CFCM de 2003 à 2005, elle est aussi experte auprès du Conseil de l'Europe. Après avoir œuvré au sein de la PJJ, elle a créé sa propre société de consulting ${ }^{39}$ sous la forme d'un cabinet privé spécialisé dans la gestion de la diversité religieuse et culturelle en entreprise. L'essentiel de ses activités consiste alors en la production de rapports, sous la forme de manuels ou de guides de la gestion de la diversité religieuse dans le secteur privé notamment, puis de guides sur la laïcité chez de grands éditeurs

36. Rapport d'information..., op. cit., p. 273.

37. Baubérot J., Bouzar D., Costa-Lascoux J., Houziaux A., Le voile, que cache-t-il ?, Paris, Éd. de l'atelier, 2004.

38. Bouzar D., Kada S., L’une voilée, l'autre pas. Le témoignage de deux femmes musulmanes françaises, Paris, Albin Michel, 2003.

39. http://www.bouzar-expertises.fr [consulté le 3 juin 2016] 
comme Hachette et Albin Michel. C'est à ce titre qu'elle devait également être sollicitée par le groupe socialiste de l'Assemblée nationale afin de produire un état des lieux des bonnes pratiques en matière de gestion de la diversité religieuse à l'échelon des communes. Ce recueil d'expériences servira notamment de trame à la publication, en décembre 2011, par la Fondation Jean Jaurès d'un Guide pratique de la laïcité sous la direction de Jean Glavany ${ }^{40}$.

Lors de son intervention devant la mission d'information parlementaire, elle a surtout développé une analyse défavorable au port du voile intégral en rattachant celui-ci au dynamisme du courant salafiste et en mobilisant un vocabulaire résolument socialement dépréciatif ("secte», "gourous », « auto-exclusion», " manipulation psychique », " endoctrinement »...) emprunté au répertoire lexical des mouvements de lutte contre les sectes et à celui de la psychologie. C'est notamment à Dounia Bouzar que l'on doit d'avoir permis aux parlementaires de recourir systématiquement au vocable de "secte» dans une acception quelque peu désociologisée pour évoquer certaines expressions littéralistes de l'islam dont le port du voile intégral serait l'emblème. Écoutons-la : « [...] alors que le mot religion vient du latin relegere et religare, c'est-à-dire "accueillir" et "relier", le mot secte signifie "suivre" et "séparer". C'est donc bien l'effet du discours qui me permet de le qualifier de sectaire : lorsque la religion provoque de l'auto-exclusion et l'exclusion des autres, on peut parler de secte. ${ }^{41}$ Le terme est en effet uniquement mobilisé comme synonyme de déviance (elle semble négliger le fait étymologique que dérivant également de sequi, la secte peut également être entendue comme simple synonyme d'école de pensée ${ }^{42}$ ), de déformation et d'auto-exclusion fondée sur une mauvaise compréhension de la religion. Cela laisse présupposer que celle qui y recourt est en mesure de savoir ce qu'est la conception normale, canonique de la religion musulmane. L'experte se transforme alors indirectement sinon en gardienne vigilante du dogme de l'islam originel, du moins d'une conception normée/normale de la religion par opposition à ce qui relèverait d'une anomalie, d'une totale hétérodoxie. « S'étonner, être choqué, dit-elle, être offensé par le niqab, c'est respecter l'islam, c'est montrer que la France n'a pas une vision archaiqque de cette religion. ${ }^{43} \mathrm{Il}$ convient donc à la fois de respecter l'islam tout en défendant

40. Glavany J., Le guide pratique de la laïcité, Paris, Fondation Jean Jaurès, 2011.

41. Rapport d'information..., op. cit., p. 274.

42. Sur les diverses acceptions et pour une analyse historique et sociologique des formes d'expressions religieuses du phénomène sectaire, V. LuCA N., Les sectes, Paris, PUF, 2004 (Que sais-je?).

43. Rapport d'information..., op. cit., p. 277. 
une vision moderne de celui-ci. D'ailleurs un parlementaire issu des rangs souverainistes de l'UMP, le député Jacques Myard, lui fera reproche de promouvoir, selon lui, un point de vue confessionnel pour s'opposer au port $\mathrm{du}$ voile intégral ${ }^{44}$. Cela provoquera un bref moment de tension durant l'audition, obligeant Dounia Bouzar à réagir et à préciser qu'elle n'entendait absolument pas défendre une approche fondée sur la foi, mais sur son expérience concrète d'anthropologue utilisant un lexique emprunté à la psychologie et la psychanalyse ${ }^{45}$.

Cette manière de penser le voile intégral comme étant hors du champ des pratiques islamiques laisse à penser qu'elle cherche à gommer le fait que cette pratique, bien que minoritaire, est pourtant bel et bien une pratique islamique. Dounia Bouzar considère que parler de dérive sectaire revient en fait à présenter le niqab pour ce qu'il est, à savoir, selon elle, un signe à la fois non religieux et une pratique étrangère à l'islam lui-même. "Mon postulat de départ et la conclusion à laquelle je souhaitais parvenir - tientelle à préciser - étaient qu'il faut appliquer le droit commun et traiter ces groupuscules comme s'ils n'étaient pas musulmans. »

Or, quand elle réduit le port du voile à une expression sectaire de l'islam (salafisme), elle tend à produire à la fois une vision assez monolithique de ce qu'est le salafisme réel, tout en suggérant en creux une vision normative de ce que devrait être l'islam. Le recours systématique, lors de son audition, au mot valise de «secte " à propos du salafisme dont le voile intégral serait l'expression pratique, devait néanmoins trouver un début de concrétisation dans la proposition $\mathrm{n}^{\circ} 11$ de la mission d'information ${ }^{46}$. Celle-ci devait en effet se fixer comme objectif de «demander à la Miviludes (Mission interministérielle de vigilance et de lutte contre les dérives sectaires) de dresser un état des lieux des éventuelles dérives sectaires qui pourraient avoir lieu

44. «[...] votre démarche qui se situe uniquement du point de vue de la foi, n'est pas sans susciter un certain malaise, particulièrement ici, à l'Assemblée nationale. [...] Au nom de quoi déciderions-nous de ce qu'est la religion? Il n'y a pas en France de ministère chargé de décider de ce que doit être la religion - car le ministère chargé des cultes les considère dans une perspective très différente. »: Rapport d'information..., op. cit., p. 279.

45. «Je suis surprise que l'on désigne mon analyse comme "religieuse", car je n'ai pas évoqué une seule fois l'islam, ni la Coran, ni Dieu. [...] Je ne parle que de la socialisation et de l'étape "psychologique" de l'endoctrinement, c'est-à-dire du discours tenue au jeune pour le mettre en situation de non-citoyenneté. Un anthropologue ne regarde pas ce que dit l'islam. » : Rapport d'information..., op. cit., p. 280.

46. Nombreuses seront les personnes auditionnées qui reprendront également à leur compte l'association voile intégral et secte, à commencer par Élisabeth Badinter. 
dans l'entourage des personnes portant le voile intégral et dont ce dernier pourrait être le révélateur ${ }^{47}$.

Cela confirme le fait que sa sollicitation en tant qu'experte en matière de voile n'avait pas vraiment pour finalité de fournir une analyse anthropologique fine de cette pratique, mais bien de conforter les réticences des parlementaires par rapport à cette pratique rigoriste en l'adossant au témoignage d'une personne médiatiquement connue en matière de gestion de la diversité religieuse et peu suspecte d'entretenir vis-à-vis de l'islam une crainte obsessionnelle. D'ailleurs, à propos du principe même de l'interdiction du voile intégral par la loi, Dounia Bouzar s'est dans un premier temps montrée plutôt réservée :

« Or toute nouvelle mesure prise contre l'islam, déclare-t-elle, offrirait une opportunité légale à tous ceux qui pratiquent la discrimination à l'égard des musulmans : que ce soit pour refuser à des femmes voilées l'accès aux banques, aux médecins ou à des lieux publics. Pour eux [les musulmans], une nouvelle loi conforterait les discriminations qui pourraient alors faire l'amalgame entre niqab et foulard voire les empêcherait de pratiquer leur religion $»^{48}$.

Elle devait finir par admettre son bien-fondé au nom du principe de sécurité. «En qualité d'éducatrice et d'anthropologue, j'estime, au contraire, très important de poser le débat plutôt sur le plan sécuritaire, comme l'a fait le Belgique. ${ }^{49}$

\subsection{L'INTERDICTION DU VOILE INTÉGRAL COMME PRÉALABLE À LA RÉFORME PAR LE HAUT DE L'ISLAM}

L'autre profil d'expert sur lequel il convient de s'arrêter est celui d'Abdennour Bidar. À l'époque de son audition, cet agrégé de philosophie, normalien, était enseignant de philosophie en classe préparatoire et l'auteur de plusieurs livres traitant des indispensables évolutions par lesquelles l'islam devait nécessairement passer pour être en harmonie totale avec le monde moderne et sortir de l'âge de la religion au profit de celui de l'humanisme ${ }^{50}$.

47. Rapport d'information..., op cit., p. 150.

48. Ibid., p. 277.

49. Ibid.

50. Un islam pour notre temps, Paris, Seuil, 2004 ; Self islam : histoire d'un islam personnel, Paris, Seuil, 2006 ; L'islam sans soumission : pour un existentialisme musulman, Paris, Albin Michel, 2008. 
Le député Gerin présentera en ces termes Abdennour Bidar : "Je suis heureux d'accueillir M. Abdennour Bidar, philosophe et professeur en classe préparatoire et à Sciences Po, qui a publié voilà quelques jours deux articles remarqués sur la question qui nous intéresse. Vous y affirmez, Monsieur Bidar, que le port du voile intégral est une innovation qui ne trouve ses racines ni dans l'histoire ni dans le Coran, et qualifiez même la burqa de "pathologie" de la culture musulmane. ${ }^{51}$ Cette présentation laisse suggérer que les parlementaires allaient pouvoir trouver dans son audition à la fois les bases d'une réfutation du voile intégral par les sources mêmes de la foi musulmane, et la réduction de ce vêtement à une expression maladive, pathologique de l'islam sui generis. Son audition a donc porté à la fois sur les fondements coraniques et sur l'étendue des prescriptions religieuses dans l'islam, ainsi que sur les nécessaires limitations à leur imposer dans une République laïque, avec comme perspective ultime la nécessité de "définir ce qu'est le bon ou le mauvais islam $»^{52}$.

Autant Dounia Bouzar s'est gardée tout au long de son audition de se référer à des textes doctrinaux musulmans ou à des spécialistes de l'islam, autant Abdennour Bidar prendra soin de légitimer son propos critique envers le voile intégral et l'orthodoxie musulmane en citant d'abord les réflexions de Gilles Kepel sur "l'islam de rupture " ${ }^{33}$, de Mohamed Arkoun pour sa description d'un islam «théologiquement protestant, mais politiquement catholique ${ }^{54}$, et enfin d'Emmanuel Lévinas pour sa réflexion sur le visage ${ }^{55}$. De même, il n'hésitera pas à renvoyer aux deux sourates du Coran (s. 24/v. 30-31 ; s. 33/v. 33) qui prescrivent à destination des femmes des règles de pudeur leur recommandant « de se couvrir afin d'éviter l'exhibition ${ }^{56}$. Ces citations coraniques devaient surtout lui permettre de mettre en tension le texte coranique qui recommande simplement d'éviter l'exhibition et ceux qui prônent la totale dissimulation du corps et du visage. Plus fondamentalement, son propos se résumera à légitimer le recours à l'interdiction législative du port du voile intégral comme un double coup d'arrêt porté au différentialisme et à la montée du radicalisme religieux.

51. Rapport d'information..., op cit., p. 285.

52. Ibid., p. 287.

53. Ibid., p. 285.

54. Ibid., p. 290.

55. Ibid., p. 286.

56. Ibid. 
«Pour travailler depuis plusieurs années sur l'islam et sur ses manifestations dans les espaces de la modernité et les sociétés multiculturelles, j'ai le sentiment - déclare Abdennour Bidar - que nous sommes confrontés à un radicalisme religieux désireux de piéger la République et la démocratie sur ses propres valeurs en cherchant à les détourner. [...] Une autre façon de nous prendre au piège de nos valeurs consiste à tenir un discours de victimisation, de stigmatisation : Vous, les Occidentaux, après avoir été colonialistes, vous faites preuve d'une incorrigible tendance à l'impérialisme, vous enfermez les minorités dans les discriminations. Et lorsque nous voulons exprimer notre différence, vous nous l'interdisez, parce que vous êtes par nature oppresseurs. Il est difficile de résister à ce procès en culpabilité qui nous est continuellement fait. ${ }^{57}$

On retrouve là l'idée selon laquelle la société française serait menacée ad intra par des opérateurs religieux radicaux suffisamment habiles pour détourner les libertés existantes au profit de la défense de particularismes, tout en instrumentalisant le registre de la culpabilité coloniale.

Il convient également de relever qu'Abdennour Bidar tend aussi à reprendre à son compte le schéma abondamment véhiculé par les tenants d'un certain féminisme conservateur, selon lequel les femmes musulmanes voilées sont systématiquement assimilées à des victimes - malgré elles - de « ceux qui leur conseillent de porter le hijab ou la burqa, afin de les instrumentaliser selon une logique d'entrisme ${ }^{58}$. Ce type de discours tend à renforcer la vision récurrente (et sociologiquement contestable) de femmes musulmanes résolument soumises et qui ne seraient pas en mesure de choisir par elles-mêmes les tenues qu'elles portent, quand bien même celles-ci (foulard simple ou niqab) ne sont pas pour autant équivalentes ni dotées de la même signification.

Enfin, Abdennour Bidar se démarque de Dounia Bouzar, lorsqu'il déclare que « le port du voile intégral est justement un problème religieux ${ }^{59}$, soulignant par-là que bien que ne renvoyant à aucune base scripturaire précise, il y aurait néanmoins hypocrisie à nier son ancrage religieux parce qu'il met en scène de façon quasi théâtrale le rapport pathologique de l'islam au formalisme et aux signes extérieurs. Ce réflexe qui consiste à vouloir aborder l'islam contemporain comme porteur d'une pathologie endogène (islamisme, salafisme...), assez fréquent chez de nombreux intellectuels de culture musulmane, contribue non seulement à essentialiser celui-ci, mais tend surtout

59. Ibid., p. 289. 
à réduire l'analyse de ces phénomènes de mutations de l'islam politique à une seule variable explicative (confusion du politique et du religieux, Coran assimilé à un code civil, ou poids du collectif sur l'individu...) et à écarter toute analyse plurifactorielle invitant à la prise en compte d'autres variables sociales et historiques (fonctionnement autoritaire des régimes dans le monde musulman, révolution démocratique confisquée, instrumentalisation permanente du religieux, corruption généralisée...)

Derrière sa dénonciation du voile intégral comme pathologie de la culture musulmane ${ }^{60}$, se niche en fait son projet intellectuel de débarrasser l'islam de ses « archaïsmes ", comme de toute forme de contrainte physique, morale ou psychique.

« [...] quel islam pouvons-nous tolérer et encadrer ? En formulant cette question, j'ai conscience qu'elle n'est peut-être pas très laïque. Pourtant, je crois qu'il faut s'en saisir. On pourrait certes nous rétorquer qu'il n'appartient pas à la République française de définir ce qu'est le bon, ou le mauvais islam. À mes yeux, cependant, une telle objection ne tient pas. Depuis des années, je défends l'idée que l'immersion des musulmans dans les sociétés occidentales représente une chance pour l'islam. Plus précisément, ce que nous imposons à l'islam au nom de nos valeurs constitue pour lui une chance de régler un certain nombre de comptes avec ses vieux démons. ${ }^{61}$

Abdennour Bidar défend l'idée que cet effort de rénovation que les musulmans ne sont pas en mesure d'impulser par eux-mêmes, de l'intérieur de leur foi, peut venir par le haut, de la loi. C'est donc elle qui fera par extension le tri entre le «bon islam » à promouvoir et à défendre et le "mauvais islam » à proscrire et à combattre.

On en vient ainsi à se demander si c'était bien le point de vue d'un philosophe familier de l'islam qui était recherché par les parlementaires, ou plutôt une caution, celle d'un musulman occidental défendant la réforme radicale de l'islam et sa réduction à une dimension culturelle émancipée de l'univers des rites et de la foi. Dès lors, on est légitimement fondé à relever que ce qui a probablement été déterminant dans son audition, c'est bien moins la dimension académique de son point de vue que sa posture de réformateur de l'islam, voire d'existentialiste musulman comme il aime à se présenter.

60. BidAR A., « La burqa, une pathologie de la culture musulmane », Libération, 29 juin 2009.

61. Rapport d'information..., op cit., p. 289. 
Dans sa stratégie de légitimation politique du principe d'interdiction du voile intégral dans l'espace public, la mission d'information sur le port du voile intégral devait rechercher des points d'appui et des relais intellectuels, notamment musulmans, sur lesquels elle pouvait adosser son intention claire de légiférer sans se voir accuser de stigmatiser une fois de plus la pratique religieuse musulmane. À défaut de pouvoir s'appuyer sur un CFCM - auditionné en sa qualité d'organe officiel représentant le culte musulman plus que jamais réticent à se prononcer unanimement en faveur d'une loi d'interdiction, la mission parlementaire devait trouver davantage de relais médiatiques et des cautions intellectuelles auprès d'experts politiquement compatibles, parce que résolument critiques vis-à-vis de cette tenue vestimentaire, et se réclamant pour l'un d'une lutte contre les déviances sectaires de l'islam, pour l'autre, fervent partisan d'une lecture résolument libérale et iconoclaste de cette religion.

\section{EN GUISE DE CONCLUSION}

Comme nous venons de le voir, il serait simpliste de considérer que la domestication de l'islam résulte exclusivement de l'action unilatérale des pouvoirs publics considérés comme une entité dotée d'une forte homogénéité, et qu'elle obéit à une simple logique d'imposition par le haut d'un schéma d'organisation préfabriqué et de pensée du «vrai islam » renvoyant à un supposé savoir administratif transcendant en la matière. Ce que par confort on a pris l'habitude de désigner comme l'« islam de France » est en fait une configuration renvoyant à des interactions multiples entre divers opérateurs publics et privés, entre des agents étatiques, des élus et des opérateurs privés musulmans, sans oublier des détenteurs de savoirs académiques et des experts familiers des médias.

En nous intéressant aux diverses modalités de légitimation sociale du vote de lois limitant le port de voiles islamiques dans la sphère publique comme dans l'espace public, notre propos ne vise pas à disqualifier en amont toute forme de débats à propos des formes d'expression de l'islam dans la société française renvoyant à des auto-compréhensions divergentes de l'islam. Loin s'en faut !

Notre objectif consiste simplement à interroger les types de sollicitation et de mobilisation de discours experts supposés rendre intelligible une situation hétérogène, afin de voir dans quelle mesure, loin de produire une vision 
dépassionnée du fait concerné comme de sa complexité, ceux-ci participent indirectement au formatage d'une version réputée autorisée de l'islam ayant droit de cité dans la République laïque.

Si le travail des parlementaires est bien de préparer des textes de loi puis de les voter, dans l'intérêt général de la collectivité nationale, il consiste aussi en produisant des normes juridiques à imposer indirectement des grilles de lecture, des analyses de phénomènes sociaux qui ne sont pas nécessairement adossées à des discours savants, ou découlant de savoirs objectivement étayés sur le domaine visé par la législation. En d'autres termes, ces normes peuvent aussi relayer des discours plus militants, c'est-à-dire socialement intéressés.

Si in fine une fois votée et promulguée, la loi doit être considérée comme la loi commune, à travers elle se lit une représentation précise, normée du social qu'elle prétend réguler, en l'occurrence une vision réputée moderne, modérée de l'islam censée avoir légitimement sa place dans l'espace public de la cité républicaine. À l'opposé, se dessine aussi en creux une vision décrite comme rétrograde et sectaire supposée illégitime de l'islam que la puissance publique entend proscrire de la cité dans l'intérêt de la société en général... et des musulmans en particulier.

Les législations adoptées à propos des différents voiles islamiques sont édifiantes parce qu'elles semblent conforter l'idée que l'islam ne saurait durablement trouver sa place légitime dans la société hexagonale sans entreprendre volontairement, par le bas, une mise au pas de certaines de ses pratiques ou de ses usages, fussent-ils le fait d'une infime minorité, donc peu représentatifs, au risque de se la voir imposer par le haut, par la loi de la République. 